This information is current as of April 25, 2023. 


\title{
Effect of CTA Tube Current on Spot Sign Detection and Accuracy for Prediction of Intracerebral Hemorrhage Expansion
}

\author{
(D) A. Morotti, (D).M. Romero, (D) M.J. Jessel, DH.B. Brouwers, (DR. Gupta, (DK. Schwab, (D)A. Vashkevich, (D)A. Ayres, (D) C.D. Anderson, \\ (D) M.E. Gurol, (D)A. Viswanathan, (D)S.M. Greenberg, (D). Rosand, and (D).N. Goldstein
}

\begin{abstract}
BACKGROUND AND PURPOSE: Reduction of CT tube current is an effective strategy to minimize radiation load. However, tube current is also a major determinant of image quality. We investigated the impact of CTA tube current on spot sign detection and diagnostic performance for intracerebral hemorrhage expansion.
\end{abstract}

MATERIALS AND METHODS: We retrospectively analyzed a prospectively collected cohort of consecutive patients with primary intracerebral hemorrhage from January 2001 to April 2015 who underwent CTA. The study population was divided into 2 groups according to the median CTA tube current level: low current $(<350 \mathrm{~mA}$ ) and high current $(\geq 350 \mathrm{~mA})$. CTA first-pass readings for spot sign presence were independently analyzed by 2 readers. Baseline and follow-up hematoma volumes were assessed by semiautomated computer-assisted volumetric analysis. Sensitivity, specificity, positive and negative predictive values, and accuracy of spot sign in predicting hematoma expansion were calculated.

RESULTS: This study included 709 patients (288 and 421 in the low- and high-current groups, respectively). A higher proportion of low-current scans identified at least 1 spot sign $(20.8 \%$ versus $14.7 \%, P=.034)$, but hematoma expansion frequency was similar in the 2 groups $(18.4 \%$ versus $16.2 \%, P=.434)$. Sensitivity and positive and negative predictive values were not significantly different between the 2 groups. Conversely, high-current scans showed superior specificity ( $91 \%$ versus $84 \%, P=.015)$ and overall accuracy $(84 \%$ versus $77 \%, P=.038)$.

CONCLUSIONS: CTA obtained at high levels of tube current showed better diagnostic accuracy for prediction of hematoma expansion by using spot sign. These findings may have implications for future studies using the CTA spot sign to predict hematoma expansion for clinical trials.

ABBREVIATIONS: $\mathrm{HmA}=$ high current; $I \mathrm{CH}=$ intracerebral hemorrhage; $\mathrm{LmA}=$ low current

$\mathbf{T}$ he CTA spot sign is a validated predictor of expansion in intracerebral hemorrhage (ICH), ${ }^{1,2}$ but the optimal acquisition protocol for spot sign identification is still unknown. There is great heterogeneity in CTA imaging parameters across centers, especially in CTA tube current, with reported milliampere ( $\mathrm{mA}$ ) values ranging from 140 to $770 .{ }^{3-7}$ Furthermore, CT is a consid-

Received January 22, 2016; accepted after revision March 17.

From the Department of Clinical and Experimental Sciences (A.M.), Neurology Clinic, University of Brescia, Brescia, Italy; Neuroradiology Service, Department of Radiology (I.M.R., R.G.), J.P. Kistler Stroke Research Center (A.M., M.J.J., K.S., A. Vashkevich, A.A., C.D.A., M.E.G., A. Viswanathan, S.M.G., J.R., J.N.G.), Division of Neurocritical Care and Emergency Neurology (J.R., J.N.G.), and Department of Emergency Medicine (J.N.G.) Massachusetts General Hospital, Harvard Medical School, Boston, Massachusetts; and Department of Neurosurgery (H.B.B.), Brain Center Rudolf Magnus, University Medical Center, Utrecht, the Netherlands.

This study was supported by grants 5R01NS073344, K23AG02872605, K23 NS086873, and R01NS059727 from the National Institute of Neurological Disorders and Stroke, a component of the National Institutes of Health.

Please address correspondence to Andrea Morotti, MD, Massachusetts General Hospital, J.P. Kistler Stroke Research Center, 175 Cambridge St, Suite 300, Boston, MA 02114; e-mail: amorotti@mgh.harvard.edu, a.morotti@ymail.com

- Indicates open access to non-subscribers at www.ajnr.org

http://dx.doi.org/10.3174/ajnr.A4810 erable source of radiation exposure, ${ }^{8}$ and concerns remain regarding minimization of radiation delivery to patients who have experienced an acute stroke. ${ }^{9}$ Tube current reduction is a common and effective strategy to minimize the global radiation exposure. ${ }^{10}$ However, this parameter is also a major determinant of image noise, and excessive reduction of the tube current level might negatively affect image quality. ${ }^{11}$ Defining the optimal CTA technical setting that predicts hematoma expansion might provide useful information for future clinical trials involving patients with ICH. Therefore, the main aim of our study was to investigate the influence of different CTA tube current levels on spot sign detection and accuracy in predicting ICH expansion.

\section{MATERIALS AND METHODS Patient Selection}

Massachusetts General Hospital institutional review board approval was received for all aspects of our study, and all the procedures comply with the Health Insurance Portability and Accountability Act. Informed written or verbal consent was obtained from patients or family members or waived by the institutional review board. We performed a single-center, retrospective analysis of a 


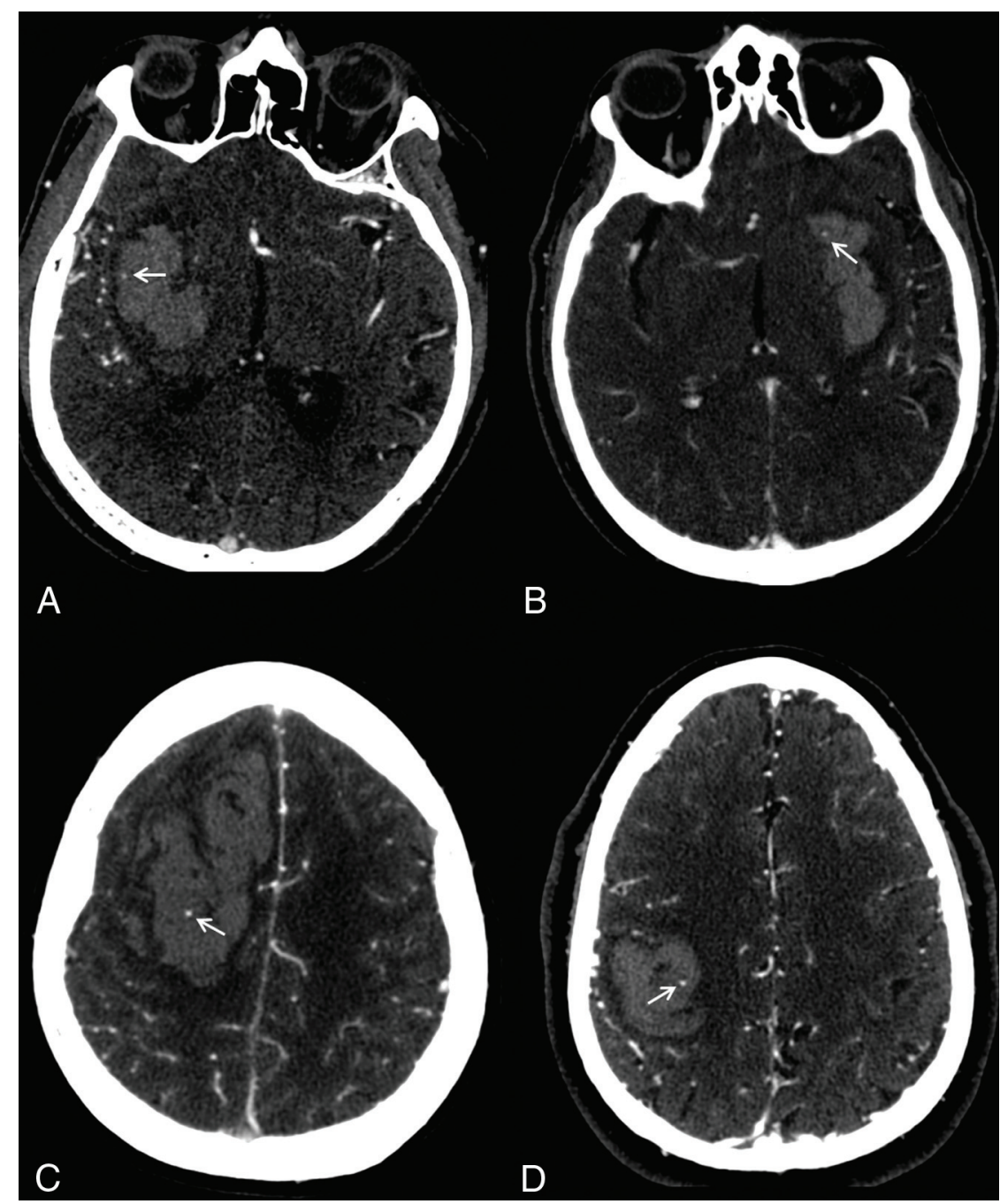

FIG 1. Appearance of the spot sign (arrows) on CTA images obtained at low tube current $(A, 170$ $\mathrm{mA} ; B, 235 \mathrm{~mA}$ ) versus high tube current $(C, 350 \mathrm{~mA} ; D, 350 \mathrm{~mA})$. All images were acquired on the same scanner at $120 \mathrm{kVp}$.

\section{Clinical Variables}

Clinical information was collected from patients, families, or the medical record and included age, sex, history of hypertension, and treatment with antithrombotic medications, including antiplatelet drugs or anticoagulant therapy. Time from symptom onset to baseline NCCT and CTA was also collected.

\section{Image Acquisition}

Axial NCCT examinations were obtained with 5-mm section thickness reconstruction. CTA was performed as part of standard clinical care by scanning from the skull base to the vertex by using an axial technique, 0.5 section pitch, $1.25-\mathrm{mm}$ collimation, and 120-140 kVp. Previous publications of an overlapping cohort described that CTA scans at our institution were typically acquired at either 235 or 350 $\mathrm{mA} .^{14,15}$ On detailed review, we found that a wide milliampere range (80-630) was used in clinical practice. Intravenous iodinated contrast material $(65-85 \mathrm{~mL})$ was administered by power injector with an infusion rate of 4-5 mL/s with SmartPrep (GE Healthcare, Milwaukee, Wisconsin), a semiautomatic contrast bolus triggering technique. The contrast materials used were Isovue 370 and Isovue 300 (Bracco, Princeton, New Jersey). Volume CT dose index ranged from 34.7-89.4 mGy (mean, 60.9; SD, 16.6) and doselength product ranged from 628.7-3763.4 $\mathrm{mGy} \times \mathrm{cm}$ (mean, 1923.6; SD, 957.5). previously described prospectively collected cohort of consecutive patients with primary $\mathrm{ICH} .{ }^{12,13}$

Patients were included if they presented from January 2001 to April 2015 with primary ICH and underwent CTA within 48 hours from symptom onset and follow-up NCCT. Patient exclusion criteria were 1) the presence of a vascular lesion or neoplastic lesion determined or suspected to be the cause of the ICH, 2) surgical evacuation of the hematoma, 3) traumatic intracranial bleeding, 4) absence of axial thin-section CTA images (section thickness, 0.625-1.25 mm), and 5) unknown CTA acquisition protocol.

Both CTA tube current and voltage are important determinants of image quality. ${ }^{11}$ However, although there is great variability in the reported current values for CTA acquisition, this is not the case for voltage. ${ }^{3-7}$ Indeed, in our cohort and in most of the previous spot sign studies, most CTA images for spot sign detection were acquired at a tube voltage level equal or above 120 $\mathrm{kVp}$ (peak). ${ }^{3-7}$ For this reason, we decided to focus our analysis on the effects of tube current on diagnostic performance. Therefore, patients with CTAs obtained at low tube voltage level $(<120 \mathrm{kVp})$ were excluded from the final analysis.

\section{Image Analysis}

The patients included in the study were divided into 2 groups: low-current $(<350 \mathrm{~mA}[\mathrm{LmA}])$ and high-current $(\geq 350 \mathrm{~mA}$ $[\mathrm{HmA}])$ scans. This cutoff was determined according to the median $\mathrm{mA}$ value. Illustrative spot sign-positive CTA images acquired at LmA versus HmA are shown in Fig 1.

Baseline NCCT scans were reviewed to determine the ICH location (deep, lobar, or infratentorial) and presence of associated intraventricular hemorrhage. Baseline and follow-up ICH volumes were calculated with semiautomated computer-assisted volumetric analysis (Analyze 11.0 software; AnalyzeDirect, Overland Park, Kansas), and hematoma expansion was defined a priori as a total volume increase greater than $6 \mathrm{~mL}$ or a relative volume increase greater than $30 \%$ from the baseline volume as previously described. ${ }^{5,16}$ For spot sign identification, first-pass CTA images were independently reviewed by 2 experienced readers (A.M., M.J.J.) blinded to CTA acquisition protocol, clinical information, and results of the follow-up NCCT. Any disagreement in reader interpretation was adjudicated by consensus agreement under the 


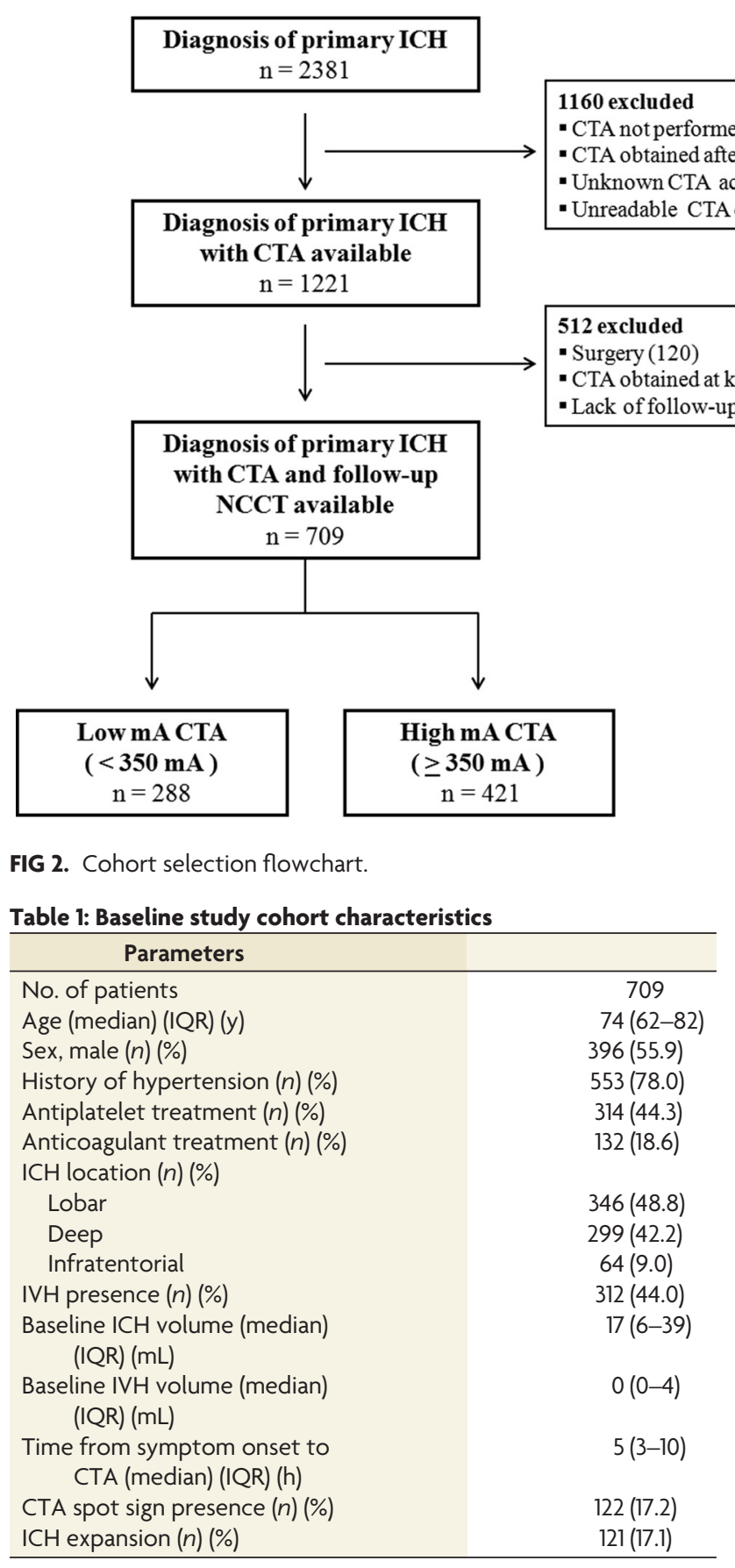

Note:- IQR indicates interquartile range; IVH, intraventricular hemorrhage.

supervision of an expert neuroradiologist (J.M.R.). Axial CTA source images were reviewed in "spot windows" (width 200, level 110) as previously described by using the following radiologic criteria for spot sign identification: 1 ) $\geq 1$ focus of contrast pooling within the $\mathrm{ICH}, 2)$ an attenuation $\geq 120 \mathrm{HU}, 3$ ) discontinuous from normal or abnormal vasculature adjacent to the hematoma, and 4) of any size and morphology. ${ }^{16}$

\section{Statistical Analysis}

All statistical analyses were performed with SPSS Version 21 (IBM, Armonk, New York). Discrete variables are summarized as count (\%). Normally distributed continuous variables are summarized as mean (SD) and continuous variables with non- normal distribution are expressed as median (interquartile range). Differences in the 2 study groups were examined with the $\chi^{2}$ test for comparison between categoric variables, $t$ test for continuous variables with normal distribution, and Mann-Whitney $U$ test for continuous variables with nonnormal distribution. Interrater and intrarater reliability for the identification of any spot sign were determined by using the Cohen $\kappa$ statistic. Subsequently, we calculated and compared sensitivity, specificity, positive predictive value, negative predictive value, and accuracy for hematoma expansion. All 95\% CIs were obtained by exact binomial methods. Comparison of the sensitivity, specificity, positive predictive value, negative predictive value, and accuracy percentages between LmA and HmA was performed by using the $\chi^{2}$ test. A $P$ value $<.05$ was considered statistically significant.

\section{RESULTS}

A total of 2381 consecutive patients with primary ICH were screened. After application of the eligibility and exclusion criteria, 709 patients were available for the analyses (Fig 2). There were 288 patients included in the LmA group and 421 included in the HmA group. The baseline characteristics of the study population are listed in Table 1. Hematoma expansion occurred in $121(17.1 \%)$ patients, and at least 1 spot sign was detected in $122(17.2 \%)$ scans. Interrater and intrarater reliability measures for spot sign detection were excellent $(\kappa=$ 0.85 and $\kappa>0.90$, respectively). Median time from symptom onset to CTA was 5 hours (interquartile range 3-10 hours). Table 2 illustrates the comparison between LmA and HmA demographic, clinical, and imaging characteristics. We observed a higher number of spot sign positive scans in the LmA group compared with the HmA group (60/288 [20.8\%] versus $62 / 421$ [14.7\%], $P=.034$ ), whereas no differences were noted in the frequency of hematoma expansion $(53 / 288$ [18.4\%] versus $68 / 421[16.2 \%], P=.434)$.

The diagnostic performance of spot sign in predicting ICH expansion stratified by tube current levels is shown in Table 3 . The LmA setting was associated with a higher frequency of false-positive cases (36/288 [12.5\%] versus 31/421 [7.4\%], $P=.022)$ and the false-negative proportion was similar between the 2 groups (29/288 [10.1\%] versus 37/421 [8.9\%], $P=.564)$. At HmA level, spot sign showed significantly superior specificity than at LmA level (91\% versus $84 \%, P=.015)$. The overall accuracy was superior in $\mathrm{HmA}$ scans ( $84 \%$ versus $77 \%, P=.038$ ).

Because there are multiple definitions of ICH expansion, we repeated the analyses using another commonly used definition: absolute growth $>12.5 \mathrm{~mL}$ or relative growth $>33 \% .{ }^{17}$ We confirmed the superior specificity (91\% versus $83 \%, P=.004)$ and 
Table 2: Patient characteristics stratified by tube current

\begin{tabular}{|c|c|c|c|}
\hline Characteristic & $\operatorname{LmA}$ & $\mathrm{HmA}$ & $P$ Value \\
\hline No. of patients & 288 & 421 & \\
\hline Age, median (IQR) (y) & $74(62-82)$ & $73(62-82)$ & .904 \\
\hline Sex, male $(n)(\%)$ & $163(56.6)$ & $233(55.3)$ & .741 \\
\hline History of hypertension (n) (\%) & $219(76.0)$ & $334(79.3)$ & .299 \\
\hline Antiplatelet treatment $(n)(\%)$ & $123(42.7)$ & $191(45.4)$ & .484 \\
\hline Anticoagulant treatment $(n)(\%)$ & $49(17.0)$ & $83(19.7)$ & .364 \\
\hline Admission INR (median) (IQR) & $1.03(1.00-1.20)$ & $1.10(1.00-1.20)$ & .331 \\
\hline $\mathrm{ICH}$ location & & & .227 \\
\hline Lobar & $130(45.1)$ & $216(51.3)$ & \\
\hline Deep & $128(44.4)$ & $171(40.6)$ & \\
\hline Infratentorial & $30(10.4)$ & $34(8.1)$ & \\
\hline IVH presence $(n)(\%)$ & $138(47.9)$ & $174(41.3)$ & .083 \\
\hline $\begin{array}{l}\text { Baseline ICH volume (median) } \\
\text { (IQR) (mL) }\end{array}$ & $18(6-46)$ & $15(6-36)$ & .018 \\
\hline $\begin{array}{l}\text { Baseline IVH volume (median) } \\
\text { (IQR) (mL) }\end{array}$ & $0(0-7)$ & $0(0-3)$ & .074 \\
\hline $\begin{array}{l}\text { Time from symptom onset to } \\
\text { CTA (median) (IQR) (h) }\end{array}$ & $5(3-10)$ & $5(3-10)$ & .342 \\
\hline CTA spot sign presence $(n)(\%)$ & $60(20.8)$ & $62(14.7)$ & .034 \\
\hline $\mathrm{ICH}$ expansion (n) (\%) & $53(18.4)$ & $68(16.2)$ & .434 \\
\hline $\mathrm{CTDI}_{\mathrm{vol}}($ mean $\pm \mathrm{SD})(\mathrm{mGy})$ & $43.3 \pm 8.9$ & $71.4 \pm 9.8$ & $<.001$ \\
\hline $\operatorname{DLP}($ mean $\pm \mathrm{SD})(\mathrm{mG} \times \times \mathrm{cm})$ & $1258.3 \pm 618.3$ & $2342.1 \pm 864.7$ & $<.001$ \\
\hline
\end{tabular}

Note:- $C T D I_{\text {vol }}$ indicates volume CT dose index; DLP, dose-length product; INR, international normalized ratio; IQR, interquartile range; IVH, intraventricular hemorrhage.

Table 3: Spot sign prediction of hematoma expansion ${ }^{\mathrm{a}}$

\begin{tabular}{lccc}
\multicolumn{1}{c}{ Variable } & LmA & HmA & $P$ Value \\
\hline No. of patients & 288 & 421 & \\
Sensitivity $(95 \% \mathrm{Cl})$ & $0.45(0.32-0.59)$ & $0.45(0.34-0.58)$ & .973 \\
Specificity $(95 \% \mathrm{Cl})$ & $0.84(0.79-0.89)$ & $0.91(0.88-0.94)$ & .015 \\
Positive predictive value $(95 \% \mathrm{Cl})$ & $0.40(0.28-0.53)$ & $0.50(0.37-0.63)$ & .267 \\
Negative predictive value $(95 \% \mathrm{Cl})$ & $0.87(0.82-0.91)$ & $0.90(0.86-0.93)$ & .367 \\
Accuracy & 0.77 & 0.84 & .038 \\
\hline
\end{tabular}

${ }^{a}$ Significant expansion was defined as $>30 \%$ or $>6 \mathrm{~mL}$ increase from baseline hematoma volume. umes. Another possible explanation is the well-known inverse relationship between image noise and CT tube current. ${ }^{10,11,22}$ Severe background noise in the LmA group might have led to detection of false spot signs because of increased graininess of the scan. Indeed, despite the higher rate of spot sign detection, the LmA setting was not associated with a significant gain in sensitivity comparing the 2 current settings. Conversely, the specificity and overall diagnostic accuracy were significantly better in the HmA group. The observed difference between the diagnostic performances of the 2 current settings may be driven by the higher frequency of false-positive cases in the LmA group. In other words, the fact that sensitivity was not affected suggests that if contrast extravasates into the hematoma, it can be successfully detected even with LmA imaging. However, HmA may optimize the ability to distinguish such contrast from natural heterogeneity of the hematoma and avoid the detection of false spot signs. It may be that dualenergy CT can help address this issue by distinguishing contrast from blood in a more robust way. ${ }^{23,24}$

Several CTA acquisition parameaccuracy ( $84 \%$ versus $76 \%, P=.008$ ) of $\mathrm{HmA}$ scans with no significant differences in sensitivity, positive predictive value, and negative predictive value (all $P$ values $>.1$ ).

\section{DISCUSSION}

This study investigated the relationship between CTA tube current, spot sign detection, and diagnostic accuracy for predicting ICH expansion. We found that the tube current level had a relevant influence on spot sign detection and diagnostic accuracy of CTA spot sign. In particular, CTA acquired with high tube current levels ( $\geq 350 \mathrm{~mA}$ ) showed higher specificity.

Our results are consistent with previous findings on the relationship between CT tube current, radiation delivery, and image quality. CTA is a commonly available tool for the emergency work-up of patients with $\mathrm{ICH}$, but additional radiation exposure is one of the main drawbacks of this technique. ${ }^{18} \mathrm{CT}$ tube current is directly associated with radiation exposure in a linear, dosedependent relationship, ${ }^{11,19}$ and as expected, we observed a significantly higher radiation dose in the HmA group. Decreasing CT tube current results in increased image noise and inferior quality of CTA images. ${ }^{19,20}$

In our study, the presence of at least 1 spot sign was significantly more frequent in the LmA group. Baseline hematoma volume is a strong predictor of spot sign presence ${ }^{21}$ and hematoma expansion. ${ }^{13}$ Therefore, this finding may simply reflect that patients in the LmA group had higher baseline ICH vol- ters can be varied to reduce the radiation dose without compromising the image quality. ${ }^{25}$ Our results suggest that if the goal of CTA is to detect spot signs, such dose reduction comes at a cost.

CTA is widely used in the work-up of $\mathrm{ICH},{ }^{26}$ and the CTA spot sign is a promising marker for early identification of patients with ICH who have the greatest opportunity to benefit from anti-expansion therapies. ${ }^{27,28}$ Therefore, patients with a false-positive spot sign may be exposed to potentially harmful anti-expansion hemostatic treatments despite having a low probability of hematoma expansion.

The only multicenter study focused on spot sign as a predictor of hematoma expansion ${ }^{1}$ had inferior diagnostic accuracy compared with single-center studies. ${ }^{5,16,17}$ Heterogeneity in the CTA acquisition protocols and image quality across various institutions might have accounted for these differences. The results of our study and the above-mentioned issues suggest the need to develop a standardized CTA acquisition protocol to optimize spot sign detection in patients with ICH.

Some limitations of the present study should be mentioned. First, this was a nonrandomized, single-center, prospective observational study with retrospective analysis of the data. In addition, the number of patients in the LmA group was relatively small. Therefore, it is best interpreted as hypothesis generating, and the findings need to be confirmed by future stud- 
ies. Second, the most relevant change in our institution's CTA protocol was the introduction of 90-second-delayed CTA images. Such images are known to capture additional spot signs, ${ }^{29}$ and it may be that the influence of current on spot sign detection is different when such images are taken into account. Third, image noise and quality were not objectively measured, so we can only speculate that image graininess and increased background noise are the mechanisms responsible for lower accuracy observed in the LmA group. Fourth, CTA tube current is not the only determinant of image quality, and other factors not considered in this study, such as different scanner models and contrast types, also may influence diagnostic accuracy. Finally, our study was designed to explore the possibility that excessive lowering of tube current reduces the diagnostic accuracy of spot sign rather than to define the optimal balance between radiation exposure, image quality, and clinical outcome. Therefore, we are not able to evaluate the clinical impact of improving CTA specificity and accuracy.

\section{CONCLUSIONS}

CTA acquisition protocol influences spot sign detection and accuracy in predicting hematoma expansion. If confirmed, our findings may have important implications for future studies using the CTA spot sign to predict hematoma expansion. Further investigations are needed to establish the optimal balance between radiation delivery, image quality, and diagnostic performance.

Disclosures: Andrea Morotti-RELATED: Grant: National Institute of Neurological Disorders and Stroke (5R01NS073344).* Anastasia Vashkevich—RELATED: Grant: National Institutes of Health. * Christopher Anderson—RELATED: Grant: National Institute of Neurological Disorders and Stroke (K23 NS086873)*; UNRELATED: Grants/ Grants Pending: National Institute of Neurological Disorders and Stroke (K23 NS086873).* Anand Viswanathan—RELATED: Grant: National Institutes of Health (R01AG047975-02, K23 AG028726-05)*; UNRELATED: Consultancy: Roche Pharmaceuticals (served on Data Safety Monitoring Board as a consultant). Jonathan Rosand-RELATED: Grant: National Institutes of Health*; UNRELATED: Grants/Grants Pending: National Institutes of Health.* Joshua Goldstein—RELATED: Grant: National Institute of Neurological Disorders and Stroke*; Support for Travel to Meetings for the Study or Other Purposes: National Institute of Neurological Disorders and Stroke*; UNRELATED: Consultancy: CSL Behring, Boehringer Ingelheim; Grants/ Grants Pending: National Institute of Neurological Disorders and Stroke.* *Money paid to the institution.

\section{REFERENCES}

1. Demchuk AM, Dowlatshahi D, Rodriguez-Luna D, et al. Prediction of haematoma growth and outcome in patients with intracerebral haemorrhage using the CT-angiography spot sign (PREDICT): a prospective observational study. Lancet Neurol 2012;11:307-14 CrossRef Medline

2. Brouwers HB, Goldstein JN, Romero JM, et al. Clinical applications of the computed tomography angiography spot sign in acute intracerebral hemorrhage: a review. Stroke 2012;43:3427-32 CrossRef Medline

3. Havsteen I, Ovesen C, Christensen AF, et al. Showing no spot sign is a strong predictor of independent living after intracerebral haemorrhage. Cerebrovasc Dis 2014;37:164-70 CrossRef Medline

4. Huynh TJ, Demchuk AM, Dowlatshahi D, et al. Spot sign number is the most important spot sign characteristic for predicting hematoma expansion using first-pass computed tomography angiography: analysis from the PREDICT study. Stroke 2013;44: 972-77 CrossRef Medline
5. Wada R, Aviv RI, Fox AJ, et al. CT angiography “spot sign” predicts hematoma expansion in acute intracerebral hemorrhage. Stroke 2007;38:1257-62 CrossRef Medline

6. Romero JM, Brouwers HB, Lu J, et al. Prospective validation of the computed tomographic angiography spot sign score for intracerebral hemorrhage. Stroke 2013;44:3097-102 CrossRef Medline

7. Goldstein JN, Fazen LE, Snider R, et al. Contrast extravasation on CT angiography predicts hematoma expansion in intracerebral hemorrhage. Neurology 2007;68:889-94 CrossRef Medline

8. Brenner DJ, Hall EJ. Computed tomography-an increasing source of radiation exposure. N Engl J Med 2007;357:2277-84 CrossRef Medline

9. Mnyusiwalla A, Aviv RI, Symons SP. Radiation dose from multidetector row CT imaging for acute stroke. Neuroradiology 2009;51: 635-40 CrossRef Medline

10. Cohnen M, Fischer H, Hamacher J, et al. CT of the head by use of reduced current and kilovoltage: relationship between image quality and dose reduction. AJNR Am J Neuroradiol 2000;21:1654-60 Medline

11. Smith AB, Dillon WP, Gould R, et al. Radiation dose-reduction strategies for neuroradiology CT protocols. AJNR Am J Neuroradiol 2007;28:1628-32 CrossRef Medline

12. Biffi A, Cortellini L, Nearnberg CM, et al. Body mass index and etiology of intracerebral hemorrhage. Stroke 2011;42:2526-30 CrossRef Medline

13. Brouwers HB, Chang Y, Falcone GJ, et al. Predicting hematoma expansion after primary intracerebral hemorrhage. JAMA Neurol 2014;71:158-64 CrossRef Medline

14. Delgado Almandoz JE, Yoo AJ, Stone MJ, et al. The spot sign score in primary intracerebral hemorrhage identifies patients at highest risk of in-hospital mortality and poor outcome among survivors. Stroke 2010;41:54-60 CrossRef Medline

15. Delgado Almandoz JE, Kelly HR, Schaefer PW, et al. CT angiography spot sign predicts in-hospital mortality in patients with secondary intracerebral hemorrhage. J Neurointerv Surg 2012;4:442-47 CrossRef Medline

16. Delgado Almandoz JE, Yoo AJ, Stone MJ, et al. Systematic characterization of the computed tomography angiography spot sign in primary intracerebral hemorrhage identifies patients at highest risk for hematoma expansion: the spot sign score. Stroke 2009;40:29943000 CrossRef Medline

17. Li N, Wang Y, Wang W, et al. Contrast extravasation on computed tomography angiography predicts clinical outcome in primary intracerebral hemorrhage: a prospective study of 139 cases. Stroke 2011;42:3441-46 CrossRef Medline

18. Macellari F, Paciaroni M, Agnelli G, et al. Neuroimaging in intracerebral hemorrhage. Stroke 2014;45:903-08 CrossRef Medline

19. Kumamaru KK, Hoppel BE, Mather RT, et al. CT angiography: current technology and clinical use. Radiol Clin North Am 2010;48: 213-35 CrossRef Medline

20. Waaijer A, Prokop M, Velthuis BK, et al. Circle of Willis at CT angiography: dose reduction and image quality-reducing tube voltage and increasing tube current settings. Radiology 2007;242: 832-39 CrossRef Medline

21. Radmanesh F, Falcone GJ, Anderson CD, et al. Risk factors for computed tomography angiography spot sign in deep and lobar intracerebral hemorrhage are shared. Stroke 2014;45:1833-35 CrossRef Medline

22. Solomon JB, Li X, Samei E. Relating noise to image quality indicators in CT examinations with tube current modulation. AJR Am J Roentgenol 2013;200:592-600 CrossRef Medline

23. Gupta R, Phan CM, Leidecker C, et al. Evaluation of dual-energy CT for differentiating intracerebral hemorrhage from iodinated contrast material staining. Radiology 2010;257:205-11 CrossRef Medline 
24. Won SY, Schlunk F, Dinkel J, et al. Imaging of contrast medium extravasation in anticoagulation-associated intracerebral hemorrhage with dual-energy computed tomography. Stroke 2013;44: 2883-90 CrossRef Medline

25. Raman SP, Mahesh M, Blasko RV, et al. CT scan parameters and radiation dose: practical advice for radiologists. J Am Coll Radiol 2013;10:840-46 CrossRef Medline

26. Khosravani H, Mayer SA, Demchuk A, et al. Emergency noninvasive angiography for acute intracerebral hemorrhage. AJNR Am J Neuroradiol 2013;34:1481-87 CrossRef

27. Goldstein J, Brouwers H, Romero J, et al. SCORE-IT: the Spot Sign score in restricting ICH growth—an Atach-II ancillary study. J Vasc Interv Neurol 2012;5:20-25

28. Meretoja A, Churilov L, Campbell BC, et al. The spot sign and tranexamic acid on preventing ICH growth-AUStralasia Trial (STOP-AUST): protocol of a phase II randomized, placebo-controlled, double-blind, multicenter trial. Int J Stroke 2014;9:519-24 CrossRef Medline

29. Ciura VA., Brouwers HB, Pizzolato R, et al. Spot sign on 90-second delayed computed tomography angiography improves sensitivity for hematoma expansion and mortality: prospective study. Stroke 2014;45:3293-97 CrossRef Medline 